\title{
Non-verbal communication in meetings of psychiatrists and patients with schizophrenia
}

Lavelle M, Dimic S, Wildgrube C, McCabe R, Priebe S. Non-verbal communication in meetings of psychiatrists and patients with schizophrenia.

Objective: Recent evidence found that patients with schizophrenia display non-verbal behaviour designed to avoid social engagement during the opening moments of their meetings with psychiatrists. This study aimed to replicate, and build on, this finding, assessing the nonverbal behaviour of patients and psychiatrists during meetings, exploring changes over time and its association with patients' symptoms and the quality of the therapeutic relationship.

Method: 40-videotaped routine out-patient consultations, involving patients with schizophrenia, were analysed. Non-verbal behaviour of patients and psychiatrists was assessed during three fixed, 2-min intervals using a modified Ethological Coding System for Interviews. Symptoms, satisfaction with communication and the quality of the therapeutic relationship were also measured.

Results: Over time, patients' non-verbal behaviour remained stable, whilst psychiatrists' flight behaviour decreased. Patients formed two groups based on their non-verbal profiles, one group $(n=25)$ displaying pro-social behaviour, inviting interaction and a second $(n=15)$ displaying flight behaviour, avoiding interaction. Psychiatrists interacting with pro-social patients displayed more pro-social behaviours $(P<0.001)$. Patients' pro-social profile was associated reduced symptom severity $(P<0.05)$, greater satisfaction with communication $(P<0.001)$ and positive therapeutic relationships $(P<0.05)$.

Conclusion: Patients' non-verbal behaviour during routine psychiatric consultations remains unchanged, and is linked to both their psychiatrist's non-verbal behaviour and the quality of the therapeutic relationship.

\author{
M. Lavelle $e^{1,2}$, S. Dimic ${ }^{1,3}$, \\ C. Wildgrube ${ }^{1,3}$, R. McCabe ${ }^{1,4}$, \\ S. Priebe ${ }^{1}$ \\ ${ }^{1}$ Unit for Social and Community Psychiatry, Queen Mary \\ University of London, London, ${ }^{2}$ Institute of Psychiatry, \\ Kings College London, London, ${ }^{3}$ East London NHS \\ Foundation Trust, London and ${ }^{4}$ University of Exeter \\ Medical School, Exeter, UK
}

This is an open access article under the terms of the Creative Commons Attribution License, which permits use, distribution and reproduction in any medium, provided the original work is properly cited.

Key words: psychiatry; therapeutic relationship; psychosis; interpersonal communication; ethological research

Mary Lavelle, Health Services and Population Research, Institute of Psychiatry, De Crespigny Park, Denmark Hill, London SE5 8AF, UK. E-mail: mary.lavelle@kcl.ac.uk

Accepted for publication June 25, 2014

\section{Significant outcomes}

- Non-verbal behaviour of patients with schizophrenia remains stable over the course of a meeting with their psychiatrist.

- Patients with schizophrenia can be classified into two groups based on their non-verbal behaviour; one group displaying non-verbal behaviour designed to avoid social interaction, and a second displaying non-verbal behaviour inviting social interaction.

- Patients' non-verbal behaviour is associated both with the behaviour of their psychiatrist and the quality of the therapeutic relationship. 


\section{Lavelle et al.}

\section{Limitations}

- A shortened version of the Ethological Coding System for Interviews was used to accommodate the quality of the naturalistic video recordings.

- As only one meeting per patient was recorded, we do not know if patients' behaviour is consistent across different meetings with the same or other psychiatrists.

- All patients were relatively stable out-patients; therefore, these results may not be generalizable to patients displaying more acute symptoms.

\section{Introduction}

The Dutch psychiatrist Rümke suggested, in 1941, that psychiatrists could intuitively and very quickly recognize a patient with schizophrenia based predominantly on the patient's non-verbal behaviour (1). This intuitive process was termed the 'praecox feeling'. Recent ethological research, analysing audio-visual recording of patients' behaviour during clinical interactions, has corroborated this and provided evidence of a link between patients' behavioural features and a diagnosis of schizophrenia. Specifically, when compared with healthy controls and patients with depression, patients with a diagnosis of schizophrenia display fewer 'pro-social' non-verbal behaviours, designed to invite and maintain social interaction such as smiling, nodding and use of hand gestures (2-5), and more 'flight' behaviours, designed to avoid social interaction, such as looking away or freezing $(6,7)$. Overall, this suggests that patients with schizophrenia can be distinguished from others through an avoidant non-verbal profile.

The subtleties of how these behaviours manifest on a more fine-grained level remains largely unexplored. Two key characteristics of non-verbal communication in schizophrenia that have yet to be investigated are (i) the variability of patients' nonverbal behaviour, both over time and between patients in this heterogeneous diagnostic group and (ii) the interpersonal aspects of patients' nonverbal communication; the influence of patients' non-verbal behaviour on those they interact with and the resulting relationships. These characteristics will form the focus of the current study.

\section{Variability of non-verbal behaviour}

A recent study has identified that participation of patients with schizophrenia during brief first social encounters increased over time (8). It is unknown whether patients' non-verbal displays also change over time. This is of particular relevance in clinical interactions, if patients' behaviour changes substantially over time it would be more difficult to assess reliably.
Schizophrenia is a particularly heterogeneous disorder (9). This raises the question as to whether the avoidant non-verbal display, identified in previous studies $(6,7)$, is pervasive across this heterogeneous patient group or rather a predominant feature of a subgroup of patients. To the authors' knowledge, only one study has investigated the prevalence of types of patients with schizophrenia based on their non-verbal behaviour during social interaction (10). Employing a pragmatic protocol, Meilijson et al. (10) classified patients as displaying appropriate or inappropriate non-verbal behaviour, based on their body posture, facial expression and gesture. Although Meilijson et al. (10) did not investigate a link with symptoms, a recent review identified that previous studies, employing similar ethological methods, have done so with mixed results (11). Where some studies have identified a link between patients' avoidant non-verbal display and their increased symptom severity overall (6, $7)$, others have associated it with patients' increased negative symptoms (12), reduced negative symptoms (3) or identified no association at all (13). This suggests that non-verbal profiles may not directly map to traditional symptom clusters. Re-classifying patients based on their objective non-verbal behaviour, rather than clinical presentation, could potentially be more sensitive to social aspects of the disorder.

\section{Interpersonal communication \& therapeutic relationship}

To date, ethological studies, investigating nonverbal behaviour in clinical interactions of patients with schizophrenia, have predominately investigated the behaviour of the patient in isolation, neglecting their partner $(2,3,5-7)$. Clinical interactions are a two-way interpersonal process, where the behaviour of both the patient and the psychiatrist influences and shapes the actions and reactions of each other $(14,15)$. Thus, analyzing the behaviour of the patient in isolation removes the context from which it occurs, limiting the inferences that can be made from such data. 
Non-verbal communication in meetings

Three ethological studies, conducted outside of a clinical setting, have investigated the behaviour of healthy control participants when interacting with a patient with schizophrenia (4, 16, 17). A common finding of these studies is that patients' partners appear to adapt their nonverbal behaviour to that of the patient, even when they are unaware of their diagnosis (4, 16). It is unclear if this pattern persists in clinical interactions where history, clinical role and relationship may influence the dynamics of the communication.

The interpersonal nature of patients' non-verbal deficits has been alluded to by Rümke (1), describing the 'praecox feeling' as manifesting in the interpersonal relationship, and experienced as a difficulty building rapport with the patient. This highlights the relevance of exploring both parties in clinical interactions. Furthermore, it may imply a relationship between non-verbal communication and the quality of the therapeutic relationship. A link between non-verbal behaviour and rapport has been consistently identified in non-clinical populations (18-21) and, more recently, non-verbal behaviour has been associated with the therapeutic relationship in psychotherapy sessions (22). A possible association between non-verbal communication and the therapeutic relationship in clinical encounters involving patients with schizophrenia has yet to be explored, but is of high clinical relevance as the quality of the therapeutic relationship has been identified as an important predictor of patients' engagement in treatment and outcome amongst patients with schizophrenia specifically (23).

\section{Aims of the study}

This study aims to replicate and build on the previous findings, employing ethological analysis to assess the non-verbal behaviour of patients and psychiatrists at three fixed, 2-min intervals during routine clinical consultations.

Four specific research questions will be addressed:

i) Does patient's non-verbal behaviour change during a consultation?

ii) Is there an association between the non-verbal behaviour of the patient and the psychiatrist?

iii) Can patient groups be identified based on their non-verbal behaviour?

iv) Is the non-verbal behaviour displayed during the consultation associated with patients' symptoms, experience of the communication and the quality of the therapeutic relationship?

\section{Material and methods}

Participants

Forty routine out-patient consultations were analysed. Each consultation involved a patient with a diagnosis of schizophrenia, or schizoaffective disorder, (according to ICD 10) and a consultant psychiatrist. Forty patients and seventeen psychiatrists participated in the consultations. All patients were between 18 and 65 years of age and, prior to the consultation, provided written informed consent for the session to be videotaped and the recordings to be used for research purposes.

\section{Procedure}

To recruit patients, eligible consecutive attenders were approached in the waiting room by an independent researcher. All consultations took place in East London and in the normal consultation offices of the consultant psychiatrists, where a videocamera was placed. All aspects of the consultation (including time, length, venue, content and style) were intended to follow the usual routine and not be influenced by the recording in any way. After the consultation, patients participated in an interview with a researcher. Structured questionnaires were administered to capture patients' sociodemographic and clinical characteristics [Positive and Negative Syndrome Scale (24)], their experience of the communication with their psychiatrist [Patient Experience Questionnaire(25)], and their rating of the therapeutic relationship [Helping Alliance Scale - client version (26)]. Psychiatrists also rated the therapeutic relationship they experienced with the patient after the clinical consultation [Helping Alliance Scale - therapist version (26)].

During the recording of the consultations, any potential interference with the normal consultation was minimized in order to capture the naturalistic interaction. As a result, participants' trunk and legs were not always visible, and the quality of the close up face varied in the video recordings. The non-verbal behaviour of the patient and psychiatrist were rated from video using a modified version of the Ethological Coding System for Interviews (27). The modified ECSI, which has been employed successfully in a previous study (6), contains 13 of the original 37 items including face, head and hand movements. Two psychiatrists (SD \& CW), not involved in the consultations, patients' care or data collection, assessed the non-verbal behaviour of patients and psychiatrists during three fixed, 2-min sections of each consultations 


\section{Lavelle et al.}

(minutes 1-3, 4-6 and 8-10). This involved psychiatrists (SD \& CW) watching silent footage of the 2 -min sections of each consultation, in 30-s intervals, to assess for the presence or absence of the behaviours.

\section{Measures}

Sociodemographic information. Patients' age, gender, employment status (unemployed or paid/selfemployment), living status (with partner or not), relationship status (significant partner or not) and illness duration (years) were assessed on a structured questionnaire.

Symptoms. The Positive and Negative Symptoms Scale (24) assessed patients' symptom severity. The PANSS is a 30 -item questionnaire measuring positive, negative and other symptom dimensions on the basis of a formal semistructured clinical interview. The scores for each symptom range from $1=$ absent to $7=$ extreme. Of the 30 items, seven are grouped to form positive symptoms, seven form negative symptoms and the remaining 16 items form a general symptom score.

Therapeutic relationship. Patients' and psychiatrists' perspectives on their experiences of the therapeutic relationship were assessed individually using the client and therapist versions of the Helping Alliance Scale (26) respectively. The Helping Alliance Scale is a self-report measure. Items on the client (patient) version inquire about patients' belief in the treatment, feeling understood and criticized, and the extent to which they feel the therapist is committed and trustworthy. Items on the therapist version include questions such as: Does the therapist get along with and look forward to meeting the patient? Do they understand the patient? Do they believe in the treatment? Responses were measured on an 11-point visual analogue scale, where $0=$ not at all and $10=$ entirely. On each questionnaire, the sum of the first five items yielded a total score, with higher scores indicating a better therapeutic relationship.

Communication. Patients' satisfaction with the communication they experienced during the consultation was assessed using the Patient Experience Questionnaire (25). Three of the five subscales (i.e., 12 questions) were used; these were 'communication experiences', 'communication barriers' and 'emotions immediately after the visit'. For the communication subscale, items were measured on a five-point Likert scale, with $1=$ disagree completely and $5=$ agree completely. The four items for the emotion scale were measured on a sevenpoint visual analogue scale, where opposing emotions were at either end. Scores were reversed so that all items measured in the same direction. Higher scores indicated greater satisfaction with communication.

Non-verbal behaviour. The videotaped non-verbal behaviour of patients and psychiatrists during the consultation was analysed using a modified version of the Ethological Coding System for Interviews (5) using a subsample of 13 behavioural aspects, including facial expressions and hand movements as has been successfully used in a previous similar study (6). The behaviours were grouped according to their meaning, resulting in seven behavioural categories: gaze (looking at their partner); gesture (hand movements during speech); pro-social behaviours (comprised of the affiliative behaviour smiling, expressing friendliness, and the submissive behaviour nodding, expressing appeasement of others to prevent hostile contact); flight behaviours (looking down, look away and freezing, signalling avoidance of social stimuli); assertion (leaning forward, signalling aggression); displacement (handface movements and fumbling, signalling tension, conflict or emotive arousal) and relaxation (fold arm across chest, laugh and neutral face, signalling low levels of emotional arousal. Each 2 min section was rated in 30-s sampling periods. The end of each 30-s period was marked by a beep, at which point researchers recorded the frequency of each behaviour within that period using the $0-1$ scoring method: 0 = behaviour not present, 1 = behaviour present at least once and $2=$ behaviour present more than once. Rare differences in the ratings between the two researchers (approximately five cases: $<1 \%$ of the total data set) were discussed to achieve a consistent rating.

To allow for comparison with previous studies, the score from each 30 -s sampling period was transformed to a zero-scoring measure $(0=$ behaviour present; 1 = behaviour present at least once). The number of 30 -s sampling periods where the behaviour was present was identified as a percentage of the total sampling periods assessed. For example, if a participant nods in two, of the four, 30 -s sampling periods, in a $2 \mathrm{~min}$ interval, they will receive a nodding score of $50 \%$ for that interval $[(2 / 4) \times 100 \%]$.

\section{Statistical analysis}

Changes in non-verbal behaviour over the three time points, for patients and psychiatrists, were assessed using a Friedman test. Spearman's 
rho correlation coefficient (2-tailed) analyses investigated the association between the nonverbal behaviour displayed by a patients and psychiatrists.

Patient groups were identified using a hierarchical cluster analysis, employing a Ward's cluster method with a squared Euclidean distance method measuring distance between cases. Through a series of steps the hierarchical cluster method combines individual cases into clusters eventually resulting in one cluster involving all cases. The relative distance between clusters informed the number of distinct clusters that were present.

Two mixed models analyses assessed for differences between clusters on (i) patients' seven nonverbal categories (e.g. pro-social, gaze etc.) and (ii) psychiatrists' seven non-verbal categories. Linear mixed models were fitted and the clustering of patients within psychiatrists was handled by including a random effect for psychiatrist.

The sociodemographic information, clinical characteristics, satisfaction with communication and therapeutic relationship scores were compared between the two patient groups using chi-squared and Mann-Whitney $U$-tests where appropriate. All analyses were performed using SPSS for Mac, version 21.0. A significance level of $P<0.05$ was set for all analyses.

\section{Results}

Of the patients who were eligible to participate, $32 \%$ did not attend their appointment, $7 \%$ were not approached (considered too ill to approach by the psychiatrist or their appointment overlapped with another study participant), 36\% refused to participate when approached and $25 \%$ consented after complete description of the study to the participants. Thus, $40 \%$ of patients approached consented to participate.

Consultations were comprised of 40 patients and 17 psychiatrists. Fifteen psychiatrists were males $(88 \%)$ and two were female; 12 were of white ethnic origin $(29 \%)$, one was black and four were Asian. Forty patients were comprised of 23 males (58\%) and 17 females all with a diagnosis of schizophrenia or schizoaffective disorder. Patients had a mean age of 45.6 years $(\mathrm{SD}=9.60$; range $=26-65), 29$ were of white ethnic origin $(73 \%)$, seven were black and four were Asian. In 20 interactions $(50 \%)$, the patient and the psychiatrist had the same ethnic background; in 20 interactions, their ethnicities differed.

Nine patients had a significant partner, whilst 31 (78\%) were single. Eighteen patients lived on their own $(45 \%), 18$ live with friends or family, and two lived in supported housing. Thirty-three patients $(83 \%)$ were unemployed and seven were in paid or self-employment. Patients' mean illness duration was 18.7 years $(\mathrm{SD}=10.8$; range $=2-41)$, with an average of 3.3 hospitalisations $\quad(\mathrm{SD}=2.9$; range $=0-14)$ with a mean total duration of 31.9 weeks $(\mathrm{SD}=38.3$; range $=0-130)$.

Patients had a mean positive symptom score of $13.6(\mathrm{SD}=6.2$; range $=7-33)$, a mean negative symptom score of $12.5 \quad(\mathrm{SD}=6.0$; range $=7-29$ ) and a mean general symptom score of $29.5 \quad(\mathrm{SD}=10.3 ; \quad$ range $=16-55)$. Patients' rating of their experience of the communication with their psychiatrist (PEC) had a mean score was 51.32 ( $\mathrm{SD}=7.89$; range $=29$ 61). Patients' evaluation of the therapeutic relationship with their psychiatrist (HAS-client version) had a mean score of $8.32(\mathrm{SD}=2.12$; range $=0.20-10)$. Psychiatrists' evaluation of the therapeutic relationship with the patient (HAStherapist version) had a mean score of 9 $(\mathrm{SD}=7.23$; range $=0.8-9)$.

\section{Non-verbal behaviour}

Change over time. The percentage of non-verbal behaviour displayed by patients and psychiatrists at time points 1,2 and 3 (i.e. minutes $1-3,4-6$ and $8-10$ respectively) are displayed in Table 1 . A Friedman test revealed that patients' nonverbal behaviour did not significantly differ between the three time points (see Table 1). Psychiatrists displayed fewer flight behaviours as the interaction progressed $(P<0.01)$, but did not significantly differ on any other behaviour. As such, the remaining analyses were conducted on the nonverbal behaviour averaged across all three-time points, which is also displayed in Table 1.

Correlation analyses. A Spearman's rho, investigating the relationship between the non-verbal behaviour displayed by patients and their psychiatrists, revealed that patients' increased pro-social display was associated with psychiatrists also displaying more pro-social behaviours (rho $(40)=$ $0.419, P=0.007$ ) and fewer flight behaviours (rho $(40)=-0.359, P=0.02)$.

Cluster analysis. Prior to performing a cluster analysis, a Spearman's rho investigated the relationship between patients' behavioural categories. Patients' displaying increased pro-social behaviour was significantly associated with patients displaying reduced flight behaviours (rho $(40)=-0.337$, $P=0.03$ ). As a result of this negative association, the variables entered into the hierarchical cluster 


\section{Lavelle et al.}

Table 1. Friedman's assessment of changes in mean percentage of patient and psychiatrists' non-verbal behaviours over time points 1-3

\begin{tabular}{|c|c|c|c|c|c|c|c|}
\hline Non-verbal behaviours & $\begin{array}{c}\text { Time } 1 \\
\text { Mean \% (SD) }\end{array}$ & $\begin{array}{c}\text { Time } 2 \\
\text { Mean \% (SD) }\end{array}$ & $\begin{array}{c}\text { Time } 3 \\
\text { Mean \% (SD) }\end{array}$ & $\chi^{2}$ & $d f$ & $P$ & $\begin{array}{c}\text { Time 1-3 } \\
\text { Mean \% (SD) }\end{array}$ \\
\hline Pat gaze & $96.25(16.55)$ & $96.25(12.07)$ & $97.50(9.47)$ & 0.33 & 2 & 0.85 & $96.96(11.29)$ \\
\hline Pat pro-social & 41.88 (18.89) & 39.53 (25.49) & $42.96(22.61)$ & 1.25 & 2 & 0.53 & $41.45(19.15)$ \\
\hline Pat flight & $22.81(18.47)$ & 19.38 (17.34) & 22.60 (18.01) & 1.79 & 2 & 0.41 & $21.59(15.01)$ \\
\hline Pat gesture & $66.25(26.88)$ & 71.25 (31.79) & $63.13(34.43)$ & 2.77 & 2 & 0.25 & $66.88(23.45)$ \\
\hline Pat displacement & $46.25(26.73)$ & $50.00(22.47)$ & 43.59 (21.72) & 4.04 & 2 & 0.13 & 46.62 (19.79) \\
\hline Pat assertion & $40.63(42.62)$ & $43.75(44.76)$ & $41.25(44.77)$ & 1.93 & 2 & 0.38 & $41.87(42.10)$ \\
\hline Pat relax & 33.31 (10.08) & $35.94(12.11)$ & $36.98(11.45)$ & 0.31 & 2 & 0.86 & 36.07 (9.61) \\
\hline Psy gaze & $98.75(5.52)$ & $100(0.00)$ & $98.13(6.67)$ & 2.80 & 2 & 0.25 & $98.96(2.79)$ \\
\hline Psy pro-social & $49.38(24.83)$ & 49.06 (18.86) & $52.81(22.02)$ & 2.77 & 2 & 0.25 & $50.42(19.10)$ \\
\hline Psy flight & $21.67(13.32)$ & $18.75(12.62)$ & $17.29(12.57)$ & 8.40 & 2 & 0.02 & 19.23 (11.14) \\
\hline Psy gesture & 57.50 (34.99) & $60.63(31.46)$ & $60.00(35.26)$ & 0.45 & 2 & 0.98 & $59.38(21.86)$ \\
\hline Psy displacement & $32.50(22.79)$ & 41.56 (28.22) & 40.63 (25.91) & 4.04 & 2 & 0.13 & $38.23(19.36)$ \\
\hline Psy assertion & 28.75 (37.36) & 33.75 (43.68) & 34.38 (39.10) & 2.49 & 2 & 0.29 & 32.29 (36.69) \\
\hline
\end{tabular}

Pat, Patient; Psy, Psychiatrist.

analyses were patients' flight and pro-social behavioural categories.

The dendrogram derived from the cluster analysis is displayed in Fig. 1. It demonstrates that two

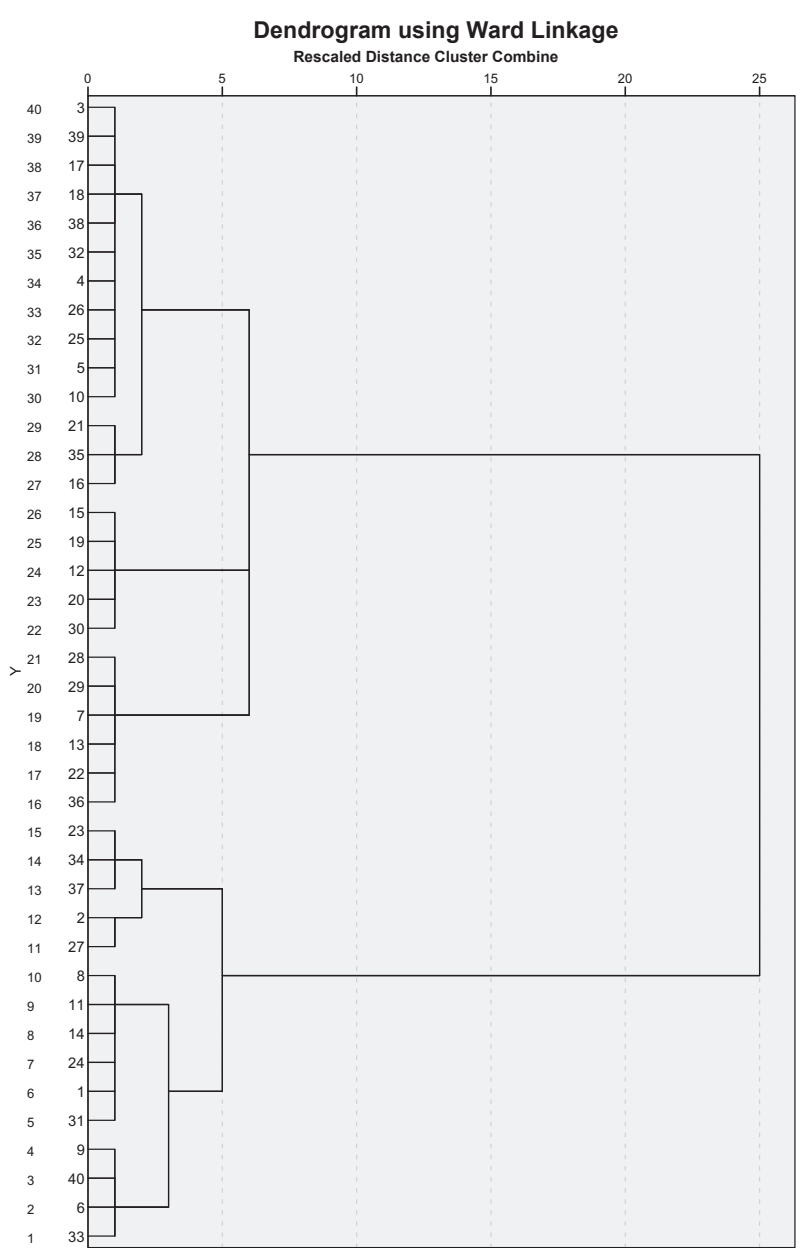

Fig. 1. Dendrogram of the two patient clusters using Ward linkage method, based on patients' displays of flight and pro-social non-verbal behaviour. clear groups can be identified, group 1 involving 25 cases and group 2 involving 15 cases. The distance between cases within each of these groups has a height of approximately 5, indicating that the grouping formed are less distinct from each other, whereas, the distance between the two groups has a height of 25 , indicating a distinct difference between them.

Mixed models analyses. A comparison of variables between patient groups (Table 2) revealed that, compared with patients' in group $2(n=15)$, patients in group $1(n=25)$ displayed significantly more pro-social behaviours $(P<0.001)$, fewer flight behaviours $(P<0.001)$. Psychiatrists also displayed more pro-social behaviour when interacting with them $(P<0.001)$. Behaviour categories of gaze, gesture, displacement, assertion and relax did not significantly differ between groups 1 and 2 for patients or psychiatrists.

Compared with patients in the flight group (group 2), patients in the pro-social group (group 1) displayed fewer positive and general symptoms $(P<0.05)$, better communication with their psychiatrist $(P<0.001)$ and better therapeutic relationship $(P<0.05$; Table 3$)$. These patients were also more likely to be employed [pro-social group: $28 \%(n=7)$ vs. flight: $0 \%]\left(\chi^{2}=5.09, P=0.03\right)$. Groups did not significantly differ in terms of age, gender, illness duration, living or relationship status.

\section{Discussion}

The current study sought to replicate the findings of previous studies investigating non-verbal behaviour of patients with schizophrenia during routine meetings with their psychiatrists. Furthermore, it 
Non-verbal communication in meetings

Table 2. Mixed models comparisons of non-verbal behaviours between patient groups

\begin{tabular}{|c|c|c|c|c|c|c|c|c|c|}
\hline \multirow[b]{2}{*}{ Model } & \multirow[b]{2}{*}{ Variables } & \multirow{2}{*}{$\begin{array}{c}\text { Group } 1(n=25) \\
M(S D)\end{array}$} & \multirow{2}{*}{$\begin{array}{c}\text { Group } 2(n=15) \\
M(S D)\end{array}$} & \multirow[b]{2}{*}{ B } & \multirow[b]{2}{*}{ SE } & \multicolumn{2}{|c|}{$95 \% \mathrm{Cl}$} & \multirow[b]{2}{*}{$\chi^{2}$} & \multirow[b]{2}{*}{$P$} \\
\hline & & & & & & Low & Up & & \\
\hline \multirow[t]{2}{*}{1} & Pat pro-social & 52.83 (12.54) & 22.50 (11.63) & 0.01 & 0.001 & 0.007 & 0.014 & 36.47 & $<0.001$ \\
\hline & Pat flight & 15.00 (11.59) & 32.59 (13.79) & -0.01 & 0.002 & -0.011 & -0.004 & 15.44 & $<0.001$ \\
\hline 2 & Psy pro-social & 56.50 (17.56) & $40.28(17.65)$ & 0.01 & 0.002 & 0.005 & 0.013 & 20.63 & $<0.001$ \\
\hline
\end{tabular}

Pat, Patient; Psy, Psychiatrist.

Model 1: Patient behaviours OIC $=15.50$

Model 2: Psychiatrist behaviours OIC $=16.18$

Table 3. Comparison of symptoms, communication satisfaction and therapeutic relationship by patient group

\begin{tabular}{|c|c|c|c|c|c|}
\hline Variable & $\begin{array}{c}\text { Group } 1 \\
(n=25) \\
M(S D)\end{array}$ & $\begin{array}{c}\text { Group } 2 \\
(n=15) \\
\mathrm{M}(\mathrm{SD})\end{array}$ & $Z$ & $d f$ & $P$ \\
\hline Positive symptoms & $12.00(7.51)$ & $16.69(7.51)$ & 2.09 & 39 & 0.04 \\
\hline General symptoms & $26.84(8.81)$ & $34.69(11.21)$ & 2.03 & 39 & 0.04 \\
\hline Negative symptoms & $11.40(5.36)$ & $14.35(6.68)$ & 1.17 & 39 & 0.25 \\
\hline $\begin{array}{l}\text { Pat - Communication } \\
\text { satisfaction }\end{array}$ & $55.00(4.83)$ & $45.00(8.25)$ & 3.59 & 39 & $<0.001$ \\
\hline $\begin{array}{l}\text { Pat - Therapeutic } \\
\text { relationship }\end{array}$ & $9.02(1.00)$ & $7.15(2.90)$ & 2.12 & 39 & 0.04 \\
\hline $\begin{array}{l}\text { Psy - Therapeutic } \\
\text { relationship }\end{array}$ & $7.53(0.96)$ & $6.70(1.89)$ & 1.46 & 39 & 0.15 \\
\hline
\end{tabular}

Pat, Patient; Psy, Psychiatrist.

aimed to build on these findings investigating the behaviour of both the patient and the psychiatrist, explore the variability and heterogeneity of patients' non-verbal behaviour and its association with symptoms and therapeutic relationship.

The results of this study reveal four main findings: First, non-verbal behaviour of patients with schizophrenia did not significantly change over the course of a clinical consultation. However, psychiatrists displayed fewer flight behaviours, designed to avoid interaction, as the consultation progressed. Second, patients' could be categorised into two groups based on their non-verbal behaviour: one group displaying predominantly pro-social behaviour designed to invite interaction (e.g. gaze, smiling, nodding) and a second displaying predominantly flight behaviour, designed to avoid interaction (e.g. gaze aversion). Third, when interacting with a patient displaying more pro-social behaviours, psychiatrists also displayed more prosocial behaviour. Fourth, patients displaying predominantly pro-social behaviour were less symptomatic, more likely to be employed and reported experiencing better quality of communication and therapeutic relationship with their psychiatrist. These findings replicate those of a previous study by Dimic et al., (6), confirming similarly high levels of flight behaviour in patients who were more symptomatic.
Variability of non-verbal behaviour

Patients' non-verbal behaviour did not significantly differ across over the course of the consultation, suggesting that patients' non-verbal profiles are relatively stable over time. Thus, employing an ethological analysis on a slice of interaction at any time point should provide a sufficiently accurate picture of the non-verbal behaviour over the course of a meeting. Since the rating of non-verbal behaviour as applied in this study is very time-consuming, the fact that an analysis of one brief period of time may provide a reasonably valid rating should facilitate further research in the field. It also makes it more likely that an impact of non-verbal behaviour on clinical outcomes can be found. Whilst patients' non-verbal displays appear to remain relatively stable over time, psychiatrists displayed fewer flight behaviours, designed to avoid social interaction, as the interaction progressed. Perhaps, this trend reflects psychiatrists' clinical sensitivity to this patient group and professional style of communication. This may be less overwhelming for patients during the opening moments of the meeting whilst still promoting engagement as the interaction progresses.

In line with previous studies $(2,3,5-7)$, a subgroup of the patients in the current study displayed an avoidant non-verbal profile, presenting with a predominance of flight behaviours designed to avoid social interaction and fewer pro-social behaviours designed to invite interaction. A commonality of previous studies was that they compared patients with schizophrenia to other groups [e.g. other patient groups $(2,6,7)$ or healthy controls $(3,5)]$ However, in the current study, variations in patients' non-verbal profiles were scrutinized on a more fine-grained level, resulting in the identification of a second patient type displaying a predominantly pro-social non-verbal profile. Employing a pragmatic protocol, Meilijson and colleagues (2004) previously identified a subgroup of patients displaying inappropriate facial expressions, posture and gesture, which impaired 


\section{Lavelle et al.}

their ability to interact with others (10). Although direct comparisons between studies are difficult due to the disparity in methods, both studies identified a subgroup of patients displaying behaviours that are not conducive to successful interaction. In the current study, this subgroup of patients were also less likely to be employed. Perhaps patients' non-verbal profiles may signal a broader social functioning impairment.

\section{Symptoms}

In line with previous studies $(6,7)$, patients who were more symptomatic displayed more flight behaviours, avoiding social interaction. Specifically, patients in the flight group, displayed more positive and general symptoms. Although previous studies have identified a link between non-verbal behaviour and patients' negative symptoms $(3,6,7,12)$ this was not identified in the current study. The variation between studies may be due, in part, to patient heterogeneity. Indeed a common feature of studies investigating non-verbal behaviour in schizophrenia is the wide non-verbal variation, with some patients displaying behaviours within healthy control ranges (11). Re-classifying patients based on their objective non-verbal behaviour rather than their clinical presentation, as has been attempted in the current study, could potentially be more sensitive in specifying clinical and biological subgroups of schizophrenia for further investigation of the disorder.

\section{Interpersonal communication}

This was the first ethological study to investigate the non-verbal behaviour of the psychiatrists alongside that of the patients during routine psychiatric consultations. Psychiatrists interacting with a patient displaying predominantly pro-social behaviour displayed a complementary pro-social pattern of behaviour. This was evident even when the clustering of patients within psychiatrists was accounted for in the analyses.

In agreement with studies conducted outside of a clinical context $(4,16,17)$, psychiatrists appear to adapt their behaviour in line with the patient. This is particularly the case when patients are displaying more pro-social behaviour. Thus, it appears that clinicians may be more likely to entrain to patients' pro-social behaviours and less likely to do so with avoidant behaviours. If further replicated, these findings may have implications for the training of mental health professionals. Clinicians should be aware of how their own behaviour can be influenced and learn to identify the important non-verbal features in patients so that they can respond in the therapeutically most helpful manner.

\section{Therapeutic relationship}

Non-verbal communication has been consistently associated with partners' evaluations of the success of the interpersonal relationship, such as rapport, both in non-clinical settings (18-21), and more recently, in clinical contexts (22). In line with this, patients displaying a pro-social profile reported a greater satisfaction with the communication during the consultation and reported a better therapeutic relationship. As the quality of the therapeutic relationships has been found to be associated with more favourable clinical outcomes in this patient group (17), the association of non-verbal behaviour and therapeutic relationships suggests that non-verbal behaviour is of clinical relevance. However, the association in this study was identified at the same time, that is, during and after one consultation. Further research may explore to what extent non-verbal behaviour is influenced by, and has an influence on, the relationship with the psychiatrist over time.

\section{Strengths and limitations}

A key strength of this study was the use of naturally occurring routine psychiatric out-patient consultations. Raters were experienced psychiatrists and researchers who were blinded towards clinical features and outcome criteria of the patients. Furthermore, this was the first study to apply the ethological coding system to both patients and psychiatrists during clinical consultations, providing a comprehensive view of the non-verbal communication between partners and what appears to be an interdependent relationship between them.

A limiting feature of this study was the fact that a shortened version of the Ethological Coding System for Interviews had to be used to accommodate the quality of the video recordings. However, nonideal recordings conditions are almost inevitable in studies of naturally occurring interactions. As this study involved out-patients, the majority of participants were taking antipsychotic medication and it is impossible to estimate the influence this may have had on their non-verbal behaviour. Future studies could investigate these characteristics in first-episode patients to address this limitation. As only one meeting for each patient was recorded, we do not know whether the behaviour is consistent across different meetings with the same or 
other psychiatrists. Furthermore, although the clustering effect of psychiatrist was accounted for in the comparison of patient groups, the limited sample size made it inappropriate to fit multilevel models to account for psychiatrist clusters in all analyses. Finally, all patients were relatively stable out-patients, and it remains to be seen whether more acute patients display similar or other types of non-verbal behaviour.

In conclusion, the results of this study have replicated, and built on, previous findings. Patients with schizophrenia can be classified into two groups based on their non-verbal profiles: one displays a predominantly pro-social profile, inviting interaction, and a second displays a predominantly flight profile, avoiding interaction. Moreover, the findings suggest that patients' non-verbal behaviours remain stable over the course of a psychiatric consultation, influence the non-verbal behaviour of their clinical partner and are associated with the quality of the therapeutic relationship. These findings highlight the stability and, particularly, the clinical relevance of non-verbal behaviour in the treatment of patients with schizophrenia.

\section{Acknowledgements}

This work was supported by the Medical Research Council (G0401323). The support of the Mental Health Research Network is gratefully acknowledged.

\section{Declaration of interest}

The authors have declared that there are no conflict of interests in relation to the subject of this study.

\section{References}

1. Rümke HC. Das Kernsyndrom der Schizophrenie und das 'Praecox-Gefuhl'. Zentralblatt gesamte Neurologie und Psychiatrie 1941;102:168-169.

2. Brüne M, Abdel-Hamid M, Sonntag C, Lehmkämper C, LANGDON R. Linking social cognition with social interaction: non-verbal expressivity, social competence and "mentalising" in patients with schizophrenia spectrum disorders. Behav Brain Funct 2009;5:6.

3. Brüne M, Sonntag C, Adbel-Hamid M, Lehmkämper C, JucKEL G, TROISI A. Nonverbal behaviour during standardized interviews in patients with schizophrenia spectrum disorders. J Nerv Ment Dis 2008;196:282-288.

4. Lavelle M, Healey PGT, McCabe R. Is nonverbal communication disrupted in interactions involving patients with schizophrenia? Schizophr Bull 2013;39:1150-1158.

5. Troisi A, Spalletta A, Pasini A. Non-verbal behaviour deficits in schizophrenia: an ethological study of drug-free patients. Acta Psychiatr Scand 1998;97:109-115.

6. Dimic S, Wildgrube C, McCabe R, Hassan I, Barnes TRE, Priebe S. Non-verbal behaviour of patients with schizophrenia in medical consultations: a comparison with depressed patients and association with symptom levels. Psychopathology 2010;43:216-222.
7. Annen S, Roser P, Brüne M. Nonverbal behavior during clinical interviews: similarities and dissimilarities among schizophrenia, mania, and depression. J Nerv Ment Dis 2012;200:26-32.

8. Lavelle M, Healey PGT, McCabe R. Participation during first social encounters in schizophrenia. PLoS ONE 2014;9:e77506.

9. Picardi A, Viroli C, Tarsitani L et al. Heterogeneity and symptom structure of schizophrenia. Psychiatry Res 2012;198:386-394.

10. Meilison SR, Kasher A, Elizur A. Language performance in chronic schizophrenia: a pragmatic approach. J Speech Lang Hear Res 2004;47:695-713.

11. Lavelle M, Healey PGT, McCabe R. Nonverbal behavior during face-to-face social interaction in schizophrenia: a review. J Nerv Ment Dis 2014;202:47-54.

12. Troisi A, Pasini A, Bersani G, Mauro MD, Ciani N. Negative symptoms and visual behavior in DSM-III-R prognostic subtypes of schizophreniform disorder. Acta Psychiatr Scand 1991;83:391-394.

13. Waters F, Rock D, Dragovic M, Jablensky A. 'Social dysmetria' in first-episode psychosis patients. Acta Psychiatr Scand 2011;123:475-484.

14. Clark HH, Schaefer EF. Contributing to discourse Cognitive Sci 1989;13:259-294.

15. Bavelas JB, Coates L, Johnson T. Listeners as co-narrators. J Pers Soc Psychol 2000;79:941-952.

16. Krause R, Steimer E, Sanger-Alt C, Wagner G. Facial expression of schizophrenic patients and their interaction partners. Psychiatry 1989;52:1-12.

17. Ellgring H. Nonverbal expression of psychological states in psychiatric patients. Eur Arch Psychiatry Clin Neurosci 1986;236:31-34.

18. Lakin JL, Chartrand TL. Using nonconscious behavioral mimicry to create affiliation and rapport. Psychol Sci 2003;14:334-339.

19. Miles LK, Nind LK, Macrae CN. The rhythm of rapport: interpersonal synchrony and social perception. J Exp Soc Psychol 2009;45:585-589.

20. Tickle-Degnen L, Rosenthal R. The nature of rapport and its nonverbal correlates. Psychol Inq 1990;1:285-293.

21. Hove MJ, Risen JL. It's all in the timing: interpersonal synchrony increases affiliation. Soc Cognition 2009;27:949960.

22. RAmSeyer F, Tschacher W. Nonverbal synchrony in psychotherapy: coordinated body movement reflects relationship quality and outcome. J Consult Clin Psychol 2011;79:284.

23. Priebe S, McCabe R. Therapeutic relationships in psychiatry: the basis of therapy or therapy in itself? Int Rev Psychiatry 2008;20:521-526.

24. KAy SR, Flszbein A, Opfer LA. The positive and negative syndrome scale (PANSS) for schizophrenia. Schizophr Bull 1987;13:261.

25. Steine S, Finset A, Laerum E. A new, brief questionnaire (PEQ) developed in primary health care for measuring patients' experience of interaction, emotion and consultation outcome. Fam Pract 2001;18:410-418.

26. Priebe $\mathrm{S}$, Gruyters $\mathrm{T}$. The role of the helping alliance in psychiatric community care: a prospective study. J Nerv Ment Dis 1993;181:552-557.

27. Troisi A. Ethological research in clinical psychiatry: the study of nonverbal behavior during interviews. Neurosci Biobehav Rev 1999;23:905-913. 